\title{
1 Plumage redness signals mitochondrial function in the House Finch
}

3 Geoffrey E. Hill ${ }^{* \dagger}$, Wendy R. Hood ${ }^{\dagger 1}$, Zhiyuan Ge ${ }^{1}$, Rhys Grinter ${ }^{2}$, Chris Greening ${ }^{2}$, James D.

4 Johnson $^{\text {', Noel R. Park }}{ }^{1,3}$, Halie A. Taylor ${ }^{1}$, Victoria A. Andreasen ${ }^{1}$, Matthew J. Powers ${ }^{1}$,

5 Nicholas M. Justyn ${ }^{1}$, Hailey A. Parry ${ }^{4}$, Andreas N. Kavazis ${ }^{4}$, Yufeng Zhang ${ }^{1,5}$

7 Affiliations:

$8 \quad{ }^{1}$ Department of Biological Sciences, Auburn University, Auburn, Alabama 36849 USA

$9{ }^{2}$ School of Biological Sciences, Monash University, Clayton, Victoria 3800, Australia

$10 \quad{ }^{3}$ Department of Molecular Biology, Princeton University, Princeton, NJ 08544

$11{ }^{4}$ School of Kinesiology, Auburn University, Auburn, Alabama 36849 USA

$12{ }^{5}$ School of Health Studies, University of Memphis, Memphis, TN 38152

13 *Correspondence to: ghill@auburn.edu

$14 \uparrow$ these authors contributed equally to this work 


\section{Abstract}

17 Carotenoid coloration is widely recognized as a signal of individual condition in various animals,

18 but despite decades of study, the mechanisms that link carotenoid coloration to condition remain unresolved. Most birds with red feathers convert yellow dietary carotenoids to red carotenoids in an oxidation process requiring the gene encoding the putative cytochrome P450 enzyme CYP2J19. Here, we tested the hypothesis that the process of carotenoid oxidation and feather pigmentation is functionally linked to mitochondrial performance. Consistent with this hypothesis, we observed high levels of red ketolated carotenoids associated with the hepatic mitochondria of molting wild house finches (Haemorhous mexicanus), and upon fractionation, we found the highest concentration of ketolated carotenoids in the inner mitochondrial membrane. We further found that the redness of growing feathers was positively related to the performance of liver mitochondria. Structural modeling of CYP2J19 supports a direct role of this protein in carotenoid ketolation that may be functionally linked to cellular respiration. These observations suggest that feather coloration serves as a signal of core functionality through inexorable links to cellular respiration in the mitochondria. 
Carotenoids are responsible for the bright red, orange, and yellow coloration of many animal species, and this coloration serves as an important social signal of individual condition $[1,2]$. In many vertebrate species, individuals that display red-shifted coloration gain a mating advantage or hold more resources [3,4], and carotenoid coloration is among the most commonly cited example of a condition-dependent sexual ornament [5-7]. Compared to animals with less red ornamentation, individuals with redder ornaments are better at resisting and recovering from parasites and managing oxidative stress, among other measures of performance [8-11]. serves as a signal of individual condition have traditionally focused on resource limitations or the need to trade carotenoid usage to support physiological functions over ornamentation [16,17], but empirical support for both of these ideas is equivocal $[12,18]$. More recently, it has been proposed that coloration is controlled by metabolic function and in turn mitochondrial efficiency $[9,19]$. In support of this idea, a recent meta-analysis revealed that the link between color expression and individual condition is strongest in bird species that rely on the metabolic

51 conversion of carotenoids for color displays [7]. Such carotenoid conversions are most relevant to red color displays because most animals that display red carotenoid coloration ingest only

53 yellow carotenoids that they oxidize to red pigments in a process that is hypothesized to take

54 place in mitochondria $[19,20]$. In this regard, it was recently shown that genes encoding proteins

55 from the cytochrome P450 monooxygenase superfamily are required for the production of red 
In this study, we explored the hypothesis that red carotenoid coloration serves as a signal

of mitochondrial performance. To do so, we compared the relationships between carotenoid coloration and mitochondrial performance in male house finches (Haemorhous mexicanus) that were actively producing ornamental feather coloration (figure 1). To produce red feather coloration used to attract females, house finches oxidize the yellow dietary carotenoid cryptoxanthin to the red pigment 3-hydroxyechinenone $(3 \mathrm{EH})$ in a process requiring the gene encoding the cytochrome P450 monooxygenase CYP2J19 [21,24]. Our central hypothesis is that the efficiency of the oxidation of yellow dietary pigments, and hence the coloration of feathers, is controlled either directly or indirectly by mitochondrial function (figure 2) [19,25]. To test this hypothesis, we performed a fractionation study to confirm the localization of red carotenoids in hepatic mitochondria. We subsequently compared several measures of mitochondrial performance in hepatic tissue of wild birds with different hues. Finally, we used this information, in conjunction with a molecular model of CYP2J19, to propose a hypothesis for how mitochondrial function may control carotenoid conversion.

\section{Materials and Methods}

\section{(a) Field Collection}

All procedures in this study were approved by the Auburn University Institutional Animal Care and Use Committee (PRN 2016-2922) and under federal (MB784373-0) and Alabama (6285940) collecting permits. We captured wild house finches at feeding stations using a walk-in basket trap as described in Hill [26]. All birds included in this study were males in the hatching year, transitioning from juvenal to first basic plumage via prebasic molt. In the juvenal plumage, house finches have no feathers with carotenoid pigmentation, so any red, orange, or 
yellow feathers in the breast plumage of birds had recently been grown. It was crucial to use birds in the process of molt and hence actively engaged in the production of red feather pigments, because this approach allowed us to match the current physiological state of birds to ornamentation that was actively being produced.

We captured birds at seven locations in Lee County, Alabama using large basket traps in which we suspended a tube feeder. Birds were captured between 07:00 and 10:00 on 11 mornings from July 20 to August 20, 2017 and 17 mornings from July 20 to August 17, 2018. Our protocol was to rapidly approach a trap holding birds - finches are undisturbed in these large traps until approached - and then to quickly move the birds from trap to brown paper bags. Paper bags enable birds to stand in a less stressful position and block all threatening visual and most auditory stimulation [26]. Thus, birds remained relatively calm between the time of capture and a maximum of three hours later when we removed them from the bag. Birds were taken from the bags, immediately anesthetized with isoflurane vapors and then sacrificed to collect tissues for physiological analyses.

(1)

\section{(b) Color analysis}

The coloration of growing breast feathers was quantified from digital images of the ventral plumage of carcasses that were taken under standardized lighting with a color standard in each image. We quantified color from digital images instead of directly from feathers using a spectrometer because some birds used in this study had grown only scattered colored feathers. The human eye could see the color of incoming feathers and digital camera images captured the coloration, but there were no colored regions large enough to allow for accurate measurement with a spectrometer. Color quantification from digital images is reliable and repeatable [27,28]. 
We used Adobe Photoshop to first standardize all images to color standards within each

104 image and then used the color sampler tool (Adobe Photoshop CS3 extended, v. 10.0, Adobe

105 Systems, San Jose, CA, USA) to quantify the hue and saturation of feathers with carotenoid

106 pigmentation at three points in each photo. Molting birds have uneven coloration and we focused

107 color quantification on the largest unbroken patches of red/orange/yellow coloration where

108 feathers were positioned to create a plane of coloration relative to the camera angle. Color

109 patches were preselected before moving the selection wand over them and then the wand was

110 moved to the center of the chosen patch and hue and saturation recorded. The PI who made the

111 color measurements was not blind to the hypotheses being tested but was blind to the

112 physiological measures for the birds being assessed; hence subconscious bias toward the

113 hypothesis could not have affected the measurements of color. We averaged the three color

114 measurements to arrive at a single hue value for each bird.

\section{(c) Tissue collection}

A total of 91 birds were used in the study. We conducted fractionation of homogenized

118 liver tissue using 55 of these birds. Fractionation of mitochondria required seven or eight times

119 the tissue mass of the average mass of a House Finch liver. Thus, the livers of seven or eight males were pooled for each fractionation analyses. We pooled all hatching year, molting males

121 captured in a particular day; hence, whether birds were added to a pool was not related to

122 coloration or other variables. We used seven birds to create a single pool during the molt window

123 of August 2017, and we used 48 birds each to create six pools, each composed of 8 birds, in the

124 summer of 2018. We increased the number of individuals pooled between years because seven 
removed from 36 additional birds (collected in July-August 2017), flash frozen in liquid nitrogen, and then stored at $-80^{\circ} \mathrm{C}$ for later analyses. The left and remaining right lobe of the liver from these birds was used for mitochondrial isolation and immediate testing of mitochondrial respiration.

\section{(d) Fractionation of mitochondria}

Within $20 \mathrm{~s}$ of dissection from carcasses, livers were rinsed and minced together in SMEE (10 mM sucrose, $250 \mathrm{mM}$ MOPS, $1 \mathrm{mM}$ EDTA, and $1 \mathrm{mM}$ EGTA) isolation buffer. During the entire procedure, all liver mitochondria preparations were kept at $4^{\circ} \mathrm{C}$. Minced livers were then homogenized with a $2 \mathrm{~mL}$ glass Teflon homogenizer at $300 \mathrm{mg}$ tissue per milliliter of buffer. Following the protocol of Trounce et al [29], as modified by Ingraham et al [29], the homogenate was centrifuged at $750 \mathrm{~g}$ for $10 \mathrm{~min}$. The supernatant was then centrifuged at 9,800 $\mathrm{g}$ for $15 \mathrm{~min}$. The resulting supernatant was saved for further separation into cytosol and microsome fractions (labeled ER-1). The microsome fraction was comprised primarily of fragments of endoplasmic reticulum (ER). The pellet was resuspended slowly by drop-wise addition of SMEE. This suspension was then centrifuged again at 9,800 $\mathrm{g}$ for $10 \mathrm{~min}$. The pellet was resuspended in SMEE buffer and layered on top of 30\% Percoll SMEE solution and subjected to ultracentrifugation at $150,000 \mathrm{~g}$ for $60 \mathrm{~min}$ along with the supernatant ER-1 from the 9,800 $g$ spin containing cytosol and microsomes. The mitochondrial-rich layer from the Percoll solution was carefully harvested and washed 3 times with centrifugation at $7000 \mathrm{~g}$ for 10 min in SMEE at $4^{\circ} \mathrm{C}$. The supernatant from ER-1 was saved as cytosol, and the pellet as microsome fraction. 
For sub-mitochondrial fractionation, we followed a method previously described by

Palczewski et al [30], with slight modification. Isolated mitochondria were diluted with SMEE to

151 concentration of $0.12 \mathrm{mg} / \mathrm{mg}$ protein. The solution was stirred on ice for $2 \mathrm{~min}$ and then diluted supernatant, which contained the outer mitochondrial membrane, was saved. The pellet, which contained mitoplast fraction, was resuspended in SMEE and then sonicated in an ice bath for 30 $\mathrm{s}$ (4 s on, $10 \mathrm{~s}$ off cycles). The sonicated material, as well as the previously saved supernatant, were then ultracentrifuged at $150,000 \mathrm{~g}$ for $60 \mathrm{~min}$ at $4^{\circ} \mathrm{C}$. inner mitochondrial membrane, while the supernatant from the same fraction contained the matrix. With this method, however, instead of having only inner mitochondrial membrane, the

160 pellet should be considered a mixture of mostly inner mitochondrial membrane and some matrix

161 content. The pellet from digitonin-treated supernatant contained outer mitochondrial membrane.

162 Using the cubes of the liver frozen previously, fractions were then verified by immunoblot 163 against subunits of carnitine palmitoyltransferase 1A (CPT1A), citrate synthase (CS) and cytochrome $c$ oxidase (COX IV). In addition to detecting ketolated carotenoids in the inner

165 mitochondrial membrane (IMM) and to lesser extent, the outer mitochondrial membrane

166 (OMM), we also detected ketolated carotenoids in layer ER-1 $\left(=3.41+1.38 \mathrm{SD} \mu \mathrm{g} \mathrm{mL} \mathrm{L}^{-1} 3 \mathrm{HE}\right)$.

167 This fraction is predicted to represent the endoplasmic reticulum-rich microsome. We did not 168 confirm the identity of this layer with a protein marker so we cannot be confident in its identity.

169 Observing ketolated carotenoids in a microsome layer would be expected if carotenoids are 
170 ketolated in or transported to the IMM because once ketolated, the pigments must be packaged

171 for transport out of hepatic cells following ketolation.

172

173

\section{(e) Mitochondria measurements}

Mitochondria were isolated following procedures outlined previously [31]. The fresh liver was minced and then homogenized in a Potter-Elvhjem PTFE pestle and glass tube. The resulting homogenate was centrifuged at $500 \mathrm{~g}$ for 10 minutes and the supernatant was then decanted through cheesecloth and centrifuged at 3,500 $\mathrm{g}$ for 10 minutes. The resulting supernatant was discarded, and the mitochondria pellet was washed in liver isolation solution twice with centrifugation at 3,500 $\mathrm{g}$ for 10 minutes. The final mitochondria pellet was suspended in a mannitol-sucrose solution.

Mitochondrial respiration was determined polarigraphically (Oxytherm, Hansatech Instruments, UK) following procedures outlined previously [31]. In one chamber, respiration was measured using $2 \mathrm{mM}$ pyruvate, $2 \mathrm{mM}$ malate, and $10 \mathrm{mM}$ glutamate as a substrate. In the second chamber, respiration was measured using $5 \mathrm{mM}$ succinate as a substrate. State 2 respiration was defined as the respiration rate in the presence of substrates, and state 3 respiration, a measure of maximal respiration, was defined as the rate of respiration following the addition of $0.25 \mathrm{mM}$ ADP to the chamber containing buffered mitochondria and respiratory substrates, and state 4 respiration was defined as the respiration rate measured after the phosphorylation of added ADP was complete. State 2, 3, and 4 respirations were measured at $40^{\circ} \mathrm{C}$ and were normalized to mitochondrial protein content. The respiratory control ratio (RCR) was calculated by dividing state 3 respiration by state 4 respiration. 
Mitochondrial membrane potential was measured as described by [32]. Briefly,

193

194

195

196

197

198

199

200

201

202

203

204

205

206

207

208

209

210

211

212

213

mitochondrial membrane potential was followed using the potential-sensitive dye safranin $\mathrm{O}$

[33]. Isolated mitochondria were incubated in standard buffer containing $3 \mathrm{mM}$ HEPES, $1 \mathrm{mM}$

EGTA, $0.3 \%(\mathrm{~W} / \mathrm{V}) \mathrm{BSA}, 1 \mu \mathrm{g} / \mathrm{ml}$ oligomycin, and $120 \mathrm{mM}$ potassium chloride $(\mathrm{pH}=7.2$ and

$40^{\circ} \mathrm{C}$ ). Mitochondria were incubated at a concentration of $0.35 \mathrm{mg} / \mathrm{ml} \mathrm{mitochondrial} \mathrm{protein} \mathrm{in}$

standard buffer with $5 \mu \mathrm{M}$ safranin $\mathrm{O}$. The change in fluorescence were measured in cuvette by

Spectramax M (Molecular Devices, Sunnyvale, CA) at an excitation of $533 \mathrm{~nm}$ and an emission

of $576 \mathrm{~nm}$. In the end of each run, membrane potential was dissipated by addition of $2 \mu \mathrm{M}$

FCCP. The relative decrease in fluorescent signal on energization of the mitochondria is used to

represent the membrane potential. Results are reported as the absolute magnitude of this change

in fluorescence, with larger changes in relative fluorescence units indicating higher membrane

potentials.

The measurement of $\mathrm{H}_{2} \mathrm{O}_{2}$ emission in isolated mitochondria was conducted using Amplex Red (Thermofisher, Waltham, MA) [31]. Formation of resorufin (Amplex Red oxidation) by $\mathrm{H}_{2} \mathrm{O}_{2}$ was measured at an excitation wavelength of $545 \mathrm{~nm}$ and an emission wavelength of $590 \mathrm{~nm}$ using a Synergy H1 Hybrid plate reader (BioTek; Winooski, VT, USA), at $40^{\circ} \mathrm{C}$ in a $96-$ well plate using succinate. To eliminate carboxylesterase interference, $100 \mu \mathrm{M}$ of phenylmethyl sulfonyl fluoride were added into experimental medium immediately prior measurement according to Miwa et al [34]. Readings of resorufin formation were recorded every 5 minutes for 15 minutes, and a slope (rate of formation) was produced from these. The obtained slope was then converted into the rate of $\mathrm{H}_{2} \mathrm{O}_{2}$ production using a standard curve and were normalized to mitochondrial protein levels. Citrate synthase activities was measured in liver 
214 homogenate as a function of the increase in absorbance from 5,5'-dithiobis-2-nitrobenzoic acid

215 reduction according to Trounce et al [29].

Western blots were conducted on liver samples to analyze a marker of lipid peroxidation

217 (4-Hydroxynonenal; 4-HNE; ab46545; Abcam, Cambridge, MA), a marker of protein oxidation

218 (protein carbonyls; OxyBlot; s7150; EMD Millipore, Billerica, MA), and a marker of

219 mitochondrial biogenesis (PGC-1 $\alpha$, GTX37356; Genetex, Irvine, CA). Each membrane was

220 stained by Ponceau S and was used as the loading and transfer control. A chemiluminescent

221 system was used to visualize marked proteins (GE Healthcare Life Sciences, Pittsburgh, PA).

222 Images were taken and analyzed with the ChemiDocIt Imaging System (UVP, LLC, Upland,

223 CA).

228 mitochondrial membrane, and matrix. The pelleted fractions were resuspended in $0.5 \mathrm{ml}$

$230250 \mu \mathrm{l}$ of ethanol, $500 \mu \mathrm{l}$ water, and $1.5 \mathrm{ml}$ of hexane:tert-Butyl ethyl ether 1:1 vol:vol. We

231 collected the organic phase from the samples, dried this under a stream of nitrogen gas, then

232 resuspended in $200 \mu \mathrm{l}$ of mobile phase. We injected $50 \mu 1$ of the resuspended extract in to an

233 Agilent 1100 series HPLC equipped with a YMC carotenoid $5.0 \mu \mathrm{m}$ column $(4.6 \mathrm{~mm} \times 250 \mathrm{~mm}$,

234 YMC). We eluted the samples with a gradient mobile phase consisting of

235 acetonitrile:methanol:dichloromethane (44:44:12) (vol:vol:vol) through 11 minutes then a ramp

236 up to acetonitrile:methanol:dichloromethane (35:35:30) from 11-21 minutes followed by 
237 isocratic conditions through 35 minutes. The column was held at $30^{\circ} \mathrm{C}$, and the flow rate was 1.2

$238 \mathrm{ml} / \mathrm{min}$ throughout the run. We monitored the samples with a photodiode array detector at 400 ,

239445 , and $480 \mathrm{~nm}$, and carotenoids were identified by comparison to authentic standards or

240 published accounts. Carotenoid concentrations were determined based on standard curves

241 established with astaxanthin (for ketocarotenoids) and zeaxanthin (for xanthophylls) standards.

\section{(g) Statistical analyses}

251 regression to evaluate this relationship. For all analyses, significance was established at $p<0.05$.

252 The stepwise model was run in SAS and the model estimated coefficients from our final model

253 were double-checked in R. In addition, we ran linear regression between variables in SAS to

254 confirm biologically relevant relationships.

\section{(h) CYP2J19 molecular modeling}

The amino acid sequence from House Finch CYP2J19 was submitted to the Robetta and 
homology modeling approach [36,37]. The highest ranked output model from each server was utilized for analysis and cross examined for consistency with the other models.

The position of the predicted heme functional group of CYP2J19 was modeled through superimposition with the structure of the nearest structurally characterized homologue (CYP2B4, PDB ID $=3$ TK3). The position of this heme group is consistent with coordination by cysteine 444 of CYP2J19. Potential substrate tunnels were identified using Caver 3.0 [38], with the Fe atom of the heme functional group set as the starting point for the search starting point. A minimum probe radius of $0.9 \AA$, a shell depth of 4 , a shell radius of 3 and a clustering depth of 3.5 was utilized. Identified tunnels were screened manually for likely involvement in substrate capture, based on size and proximity of the tunnel exit to the putative lipid embedded portion of CYP2J19. To validate the selected substrate tunnel, its position was compared to ligands in previous solved cytochrome P450 structures (PDB codes utilized: 4H1N, 3C6G, 4UFG, 3EBS, 1R90, 4I8V, 5X24, 4R20, 5T6Q, 3UA1, 2Q9F, 1ZOA, 4KEY, 2UWH and 4KPA). P450s bound to compact ligands accommodated them in a distinct region below the heme ligand, while elongated substrates clustered on the opposite side. The substrate tunnel predicted in CYP2J19 corresponded to the position of these larger substrates, which is consistent with the elongated nature of the carotenoid substrates.

In order to determine the likelihood that the N-terminus of CYP2J19 forms a lipid anchor, we analyzed the primary amino acid sequence and found that, typical of lipid anchors and secretion signal peptides, the first 45 amino acids are composed of predominantly hydrophobic residues. Both modelling programs, as well as the secondary structure prediction program JPRED, predicted that the majority of this region is $\alpha$-helix in structure. This suggests 
that the N-terminus of CYP2J19 indeed forms a lipid anchoring helix. The models and associated analysis are contained in supplemental files associated with the manuscript.

\section{Results and discussion}

(a) Red carotenoids are localized in the inner mitochondrial membrane in molting house finches

A key prediction of the hypothesis that carotenoid coloration is linked to cellular respiration is that red carotenoid pigments should be present within mitochondria [19,25]. We previously documented that high levels of red ketolated carotenoids are associated with mitochondria in the livers of molting house finches [39], but we could not rule out that these pigments were actually concentrated in external mitochondrial-associated membranes. To resolve this, we isolated and fractionated hepatic mitochondria into IMM, OMM, and matrix, and subsequently measured the concentrations of the red carotenoid $3 \mathrm{HE}$ in the fractions.

Protein markers confirmed the separation of mitochondrial components (figure 3C). Consistent with our hypothesis, $3 \mathrm{HE}$ was present in high concentrations in the IMM $(2.19 \mu \mathrm{g}$ $\mathrm{mL}^{-1}$ ) (figure 3A and 3B). These levels were significantly higher than in the matrix (by 22-fold; $p=0.009$ ) and OMM (by 7.8-fold; $p=0.014$ ) fractions. The high levels of $3 \mathrm{HE}$ in the IMM fraction of mitochondria is strong evidence that ketolated carotenoids are not merely associated with mitochondria, but instead abundant in the interior membrane. This observation is of potentially great significance for understanding the mechanisms that underlie honest signaling via red carotenoid coloration. Given carotenoids compounds are co-localized with the electron 
transport system in the IMM, they are ideally localized within the cells of house finches to signal respiratory performance during sexual displays. These findings contrast with mammals, which do not use ketolated carotenoids in social signaling [42]; most mammals use enzymatic mechanisms to specifically exclude carotenoids from the mitochondria, including carotenoidcleaving enzyme $\beta$, $\beta$-carotene-9,10-dioxygenase (BCO2) [41], and murine knockdowns of this gene have negative consequences for physiological function [40].

(b) Carotenoid coloration is strongly correlated with mitochondrial function in house finches

The second key prediction of our central hypothesis is that bird coloration is positively associated with mitochondrial bioenergetic capacity $[9,25]$. We tested this prediction by capturing wild house finches that were actively growing their red feathers, quantifying the coloration of growing feathers, and measuring the performance of functional hepatic mitochondria. We measured isolated liver mitochondrial state 2 (proton leak), 3, and 4 respiration rates, $\mathrm{RCR}$, and mitochondrial membrane potential both in the presence of OXPHOS complex I substrates (pyruvate, malate, and glutamate) and OXPHOS complex II substrate (succinate). We also quantified isolated liver mitochondrial $\mathrm{H}_{2} \mathrm{O}_{2}$ production and whole liver tissue adduct levels of 4-hydroxynonenal (4-HNE; a by-product of lipid peroxidation), protein carbonyls, citrate synthase activity, and PGC-1 $\alpha$ protein levels (the mitochondrial biogenesis transcriptional activator).

We ran a stepwise-backwards regression model to determine which of the 15 variables made a significant contribution to the coloration of growing feathers. Six variables contributed to the best fit statistical model $\left(\mathrm{F}=10.6, \mathrm{df}=6,25, p<0.001, \mathrm{R}^{2}=0.727\right)$; $\mathrm{RCR}$, state 2 respiration, 
state 4 respiration, and mitochondrial membrane potential with complex I substrates; 4-HNE levels and PGC-1 $\alpha$ protein levels (figure 4, table 1). The strongest associations in both the backward regression model and the full multiple regression model were between the redness of growing feathers and both RCR and PGC-1 $\alpha$ (figure 4).

The ten reddest birds had an average RCR 1.7-fold greater than the ten yellowest individuals $(p<0.001)$. The RCR is a measure of mitochondrial coupling efficiency; low ratios are associated not only with reduced performance but also with disease and aging in birds and mammals $[45,46]$. RCR is a ratio of state 3 divided by state 4 respiration, thus an increase in RCR should be associated with an increase in state 3 (maximum respiratory performance), a decrease in state 4 (basal, non-phosphating, respiratory performance), or both. In this study, RCR was greater in redder birds and, counter-intuitively, state 4 respiration was also higher in redder birds than dull birds in the backward-regression, but not full model. Given the inconsistency of the full and backward regression models, we interpret the state 4 values relative to RCR with caution. A direct comparison of the linear relationship between RCR and both state 3 and state 4 respiration is more revealing and clearly suggests that higher RCR is associated with lower state 4 respiration (linear regression, $\mathrm{F}_{31}=45.8, p<0.001$ ) but not higher state 3 respiration (linear regression, $\left.\mathrm{F}_{31}=0.39, p=0.53\right)$. This indicates that the hepatic mitochondria of redder birds appear to have lower costs associated with supporting basal respiratory than duller birds. Further, red birds also had increased mitochondrial membrane potential with complex I in both regression models and less leak (state 2 respiration) in the backward regression model and a near significant trend in the full model (table 1). These findings are also consistent with higher coupling efficiency associated with red coloration and supports the prediction that red birds have better mitochondrial function than drab birds. 

improved ability to combat disease relative to dull birds $[52,53]$.

\section{(c) An enzymatic model for how carotenoid coloration may be controlled by}

\section{mitochondrial bioenergetics}


$3 \mathrm{EH}$ is localized. One way in which these processes may be linked is through the putative cytochrome P450 enzyme CYP2J19, which has been shown to genetically required for the production of red carotenoid pigments in birds and turtles [21,22,57]. Based on this genetic link we hypothesized that this protein is an enzyme that catalyzes ketolation of yellow dietary carotenoids to their red derivatives in a reaction requiring an electron donor (e.g. NAD(P)H) and oxygen [21] (figure 2). This requirement for reducing equivalents produced in the mitochondria for carotenoid conversion could serve as a link between mitochondrial function and pigmentation.

As an initial exploration of this hypothesis and to demonstrate the plausibility of a mechanistic link between the ketolation of carotenoid pigments and aerobic respiration in cells, we used an in silico approach to create a molecular model of CYP2J19 (figure 5A, movie S1). This model strongly suggests that, like many other cytochrome P450s, CYP2J19 possesses an Nterminal anchor tethering it to a cellular membrane (figure 5A). As carotenoids are lipophilic, this localization would position CYP2J19 ideally for performing the ketolation reaction. We identified a substrate binding channel in CYP2J19 leading to its heme cofactor (figure 5A,C). This tunnel emerges from the region of CYP2J19 predicted to be embedded in the lipid bilayer, based on analysis of membrane-associated cytochrome P450 enzymes [58,59]. It is lined by largely hydrophobic residues and is of dimensions compatible with carotenoid binding (figure 5B,C). Each of these structural characteristics of CYP2J19 support the hypothesis that it catalyzes the conversion of yellow dietary carotenoids to red ketolated carotenoids in birds. In turn, these characteristics of CYP2J19 support the hypothesis that its activity could directly link mitochondrial performance with carotenoid ketolation (figure 5D). Several scenarios are possible. At present, it is unknown whether or not CYP2J19 localizes within 
mitochondria, but if CYP2J19 is anchored to the IMM, then its catalytic function would be affected by various aspects of mitochondrial performance; the enzyme would be particularly sensitive to mitochondrial redox state and would share an electron carrier with other key mitochondrial enzymes if it is NADH-dependent (i.e. complex I) or NADPH-dependent (e.g. thioredoxin or glutathione reductases) (figure 5D). Alternatively, yellow carotenoids may be oxidized by CYP2J19 in the ER before being imported into the IMM or, as suggested previously [25], may be modified due to respiratory activity or ROS production independently of CYP2J19. Future experiments should focus on determining the localization of CYP2J19, the biochemical role it plays in carotenoid ketolation, and how its activity responds to aerobic respiration.

\section{Conclusions}

In this work, we demonstrate that (i) red carotenoids are localized in the inner mitochondrial membrane and (ii) plumage coloration is strongly correlated with mitochondrial function. Thus, we conclude that plumage coloration signals mitochondrial function, and hence core cellular functionality, in the house finch. Moreover, we propose the hypothesis that mitochondrial activity directly controls carotenoid ketolation. Detailed biochemical studies are needed to further test this latter hypothesis and it remains plausible that mitochondrial activity indirectly rather than directly controls carotenoid oxidation. The strength of the correlations between plumage coloration and mitochondrial function, the co-localization of the red carotenoids and the mitochondrial respiratory chain, and the molecular models predicting membrane localization and carotenoid compatibility of CYP2J19 all support a functional link between aerobic respiration in mitochondria and production of red ornamental coloration. 
419 solution to a long-standing puzzle in evolutionary and behavioral biology: what maintains the

420 honesty of signals of individual condition? [13]. Carotenoid coloration has been documented to

421 signal a wide range of measures of individual performance, such as foraging ability, overwinter

422 survival, immune system function, predator avoidance, and cognition $[3,5]$. In turn,

423 mitochondrial function is a critical component of these same processes. Linking the

424 ornamentation used in mate choice to function of core respiratory processes provides a novel

425 mechanistic explanation for why carotenoid coloration relates to a range of aspects of individual

426 performance and why females use plumage redness as a key criterion in choosing mates. 
437

438

439

440

441

442

443

444

445

446

447

448

449

450

451

452

453

454

455

456

457

458

\section{Funding}

Lab work was supported by NSF grants IOS1453784 and OIA1736150 to W.R.H.. R.G. was supported by a Sir Henry Wellcome Postdoctoral Fellowship (106077/Z/14/Z) and C.G. was supported by an ARC DECRA Fellowship (DE170100310).

\section{Authors contributions}

G.E.H. contributed to conceptualization, visualization, supervision, writing, and editing. W.R.H. contributed to conceptualization, visualization, supervision, funding acquisition, writing, and editing. J.D.J. contributed to conceptualization and editing. Z.G., N.R.P., H.A.T., V.A.A., M.J.P., N.M.J, and H.A.P. contributed to investigation and editing. R.G. and C.G. contributed to methodology, investigation, visualization, writing, and editing. A.N.K. contributed to methodology, supervision, writing, and editing. Y.Z. contributed to conceptualization, methodology, investigation, data curation, visualization, formal analysis, writing, and editing.

\section{Competing interests}

Authors have no competing interests.

\section{Data accessibility}

All data are available from the Dryad database (will be submitted after manuscript is accepted, accession number TBA).

\section{Acknowledgments}

Maxwell Williams and Jordan Marquez assisted with trapping and laboratory assays. Matthew Toomey and Joseph Corbo conducted carotenoid analysis of mitochondrial fractions. Ryan

Weaver, Chloe Josefson, and Kyle Heine provide valuable discussion and feedback. Vincent Careau suggested how to present the results of the backward regression model. 
Figure 1. Hatching year house finches undergoing a first pre-basic molt, similar to those used in this study. The bird on the left is similar in hue to the drabbest male included in this study while the bird on the right is similar in hue to the brightest male included.

Figure 2. A schematic summary of the hypothesized links between red feather coloration and mitochondrial function. To produce red feathers, house finches ingest the yellow carotenoid cryptoxanthin and oxidize it to the red pigment 3-hydroxyechinenone. We hypothesized that ketolation efficiency is linked to mitochondrial bioenergetics. By this hypothesis, birds with low mitochondrial function have limited ketolation capacity and produce yellow feathers. Birds with high mitochondrial function have improved ketolation capacity and produce red feathers.

Figure 3. Ketolated carotenoid content of mitochondrial fractions from the livers of molting

473 house finches. (A) The concentration of the red carotenoid 3-hydroxyechinenone (3HE) in each

474 of three fractions of mitochondria isolated from house finch livers. 3HE concentration is higher

475 in the inner mitochondrial membrane (IMM) than the matrix or outer mitochondrial membrane

476 (OMM). (B) The relative 3HE content of each fraction was also evident in the color of fractions.

478 synthase (CS), a citric acid enzyme located primarily in the matrix that also binds to the IMM,

479 cytochrome $c$ oxidase (COX IV), a protein of the electron transport system in IMM, and

480 carnitine palmitoyltransferase I (CPT1), an enzyme that is unique to the OMM. Different lower-

481 case letters above the data indicate significant differences among treatment groups $(p<0.05)$. 
482 Standard deviation bars are given. Western blots from which protein concentrations were

483 estimated are given.

484

Figure 4. The relationships between feather redness and mitochondrial energy capacity and

486

487

488

489

490

491

492

493

494

495

496

497

498

499

500

501

502

503

504 biogenesis. The results of a backward stepwise regression analysis $(\mathrm{F}=10.6, \mathrm{df}=6,25, p<$

$\left.0.001, \mathrm{R}^{2}=0.727\right)$ suggest that redness was most strongly correlated with $(\mathbf{A})$ respiratory control

ratio $(\mathrm{P}<0.001)$ and $(\mathbf{B})$ PGC-1 $\alpha$ protein levels $(\mathrm{P}<0.001)$. Results of the full model and

backwards regression are given in table 1.

Figure 5. A possible molecular mechanism for the ketolation of carotenoids in the inner mitochondrial membrane. (A) A molecular model of the cytochrome P450 monooxygenase CYP2J19. Left: Cartoon ribbons colored from blue (N-terminal) to red (C-terminal), heme cofactor in black, and predicted N-terminal lipid anchor in blue. Right: Same model colored white with substrate tunnel shown as green spheres (right). (B) The length of the CYP2J19 substrate binding tunnel (left) is comparable to that of the putative carotenoid substrate cryptoxanthin (right). (C) The predicted substrate tunnel in CYP2J19 is surrounded by predominantly hydrophobic amino acids sidechains. Side chains surrounding tunnel are shown as sticks with representative hydrophobic residues labelled. The predicted CYP2J19 substrate tunnel (left) corresponds to the position of large substrates in previously solved cytochrome P450 structures (right). (D) A model for how the putative ketolation reaction performed by CYP2J19 may link red coloration to respiratory performance. 
bioRxiv preprint doi: https://doi.org/10.1101/728873; this version posted August 8, 2019. The copyright holder for this preprint (which was not certified by peer review) is the author/funder, who has granted bioRxiv a license to display the preprint in perpetuity. It is made available under aCC-BY-NC-ND 4.0 International license.

\section{Fig 1}

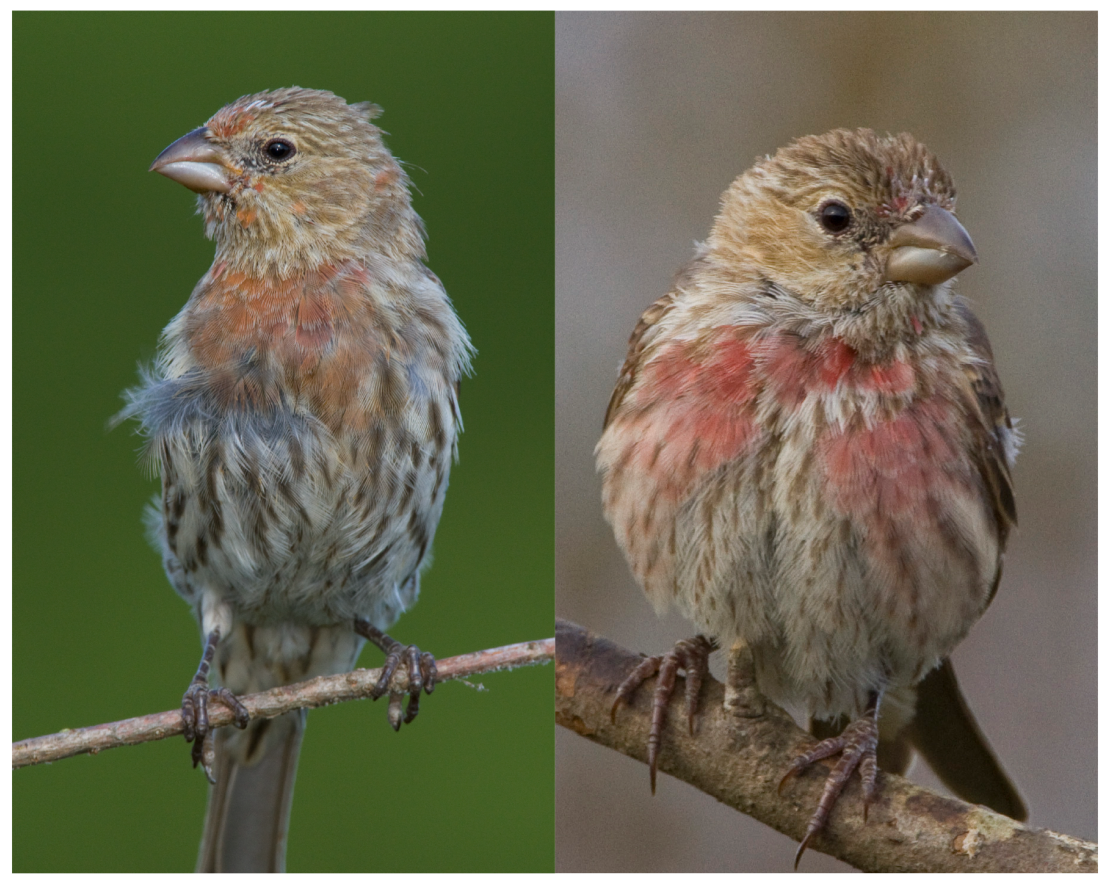



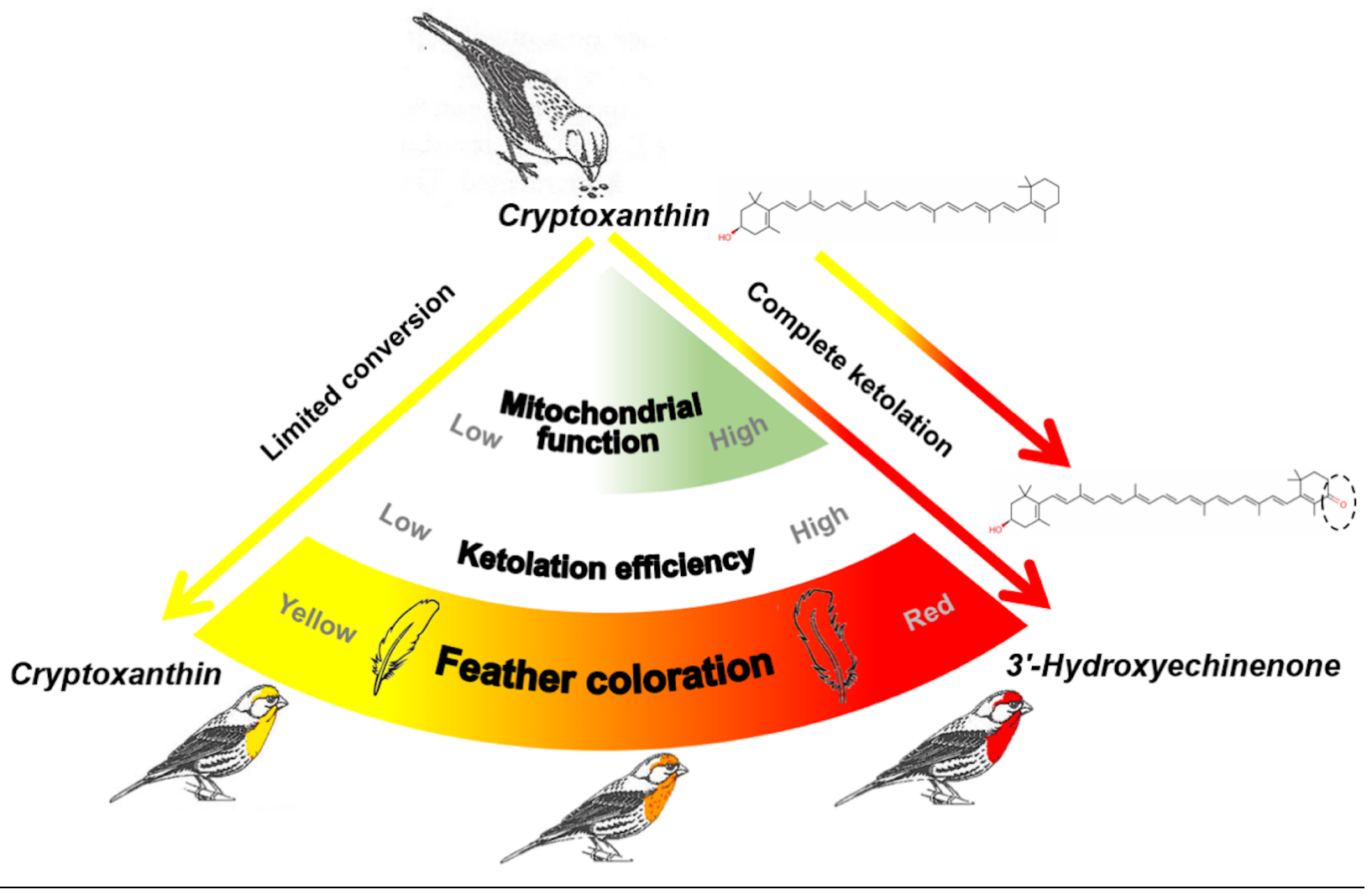

510

511 
A

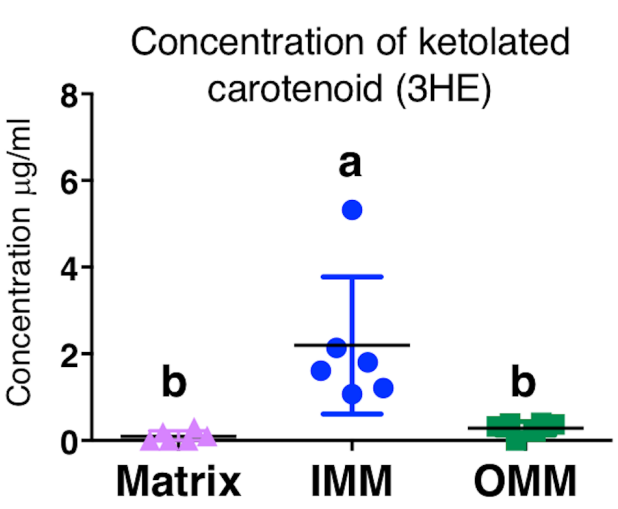

B
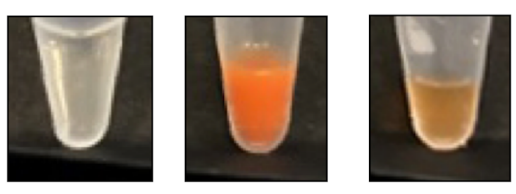
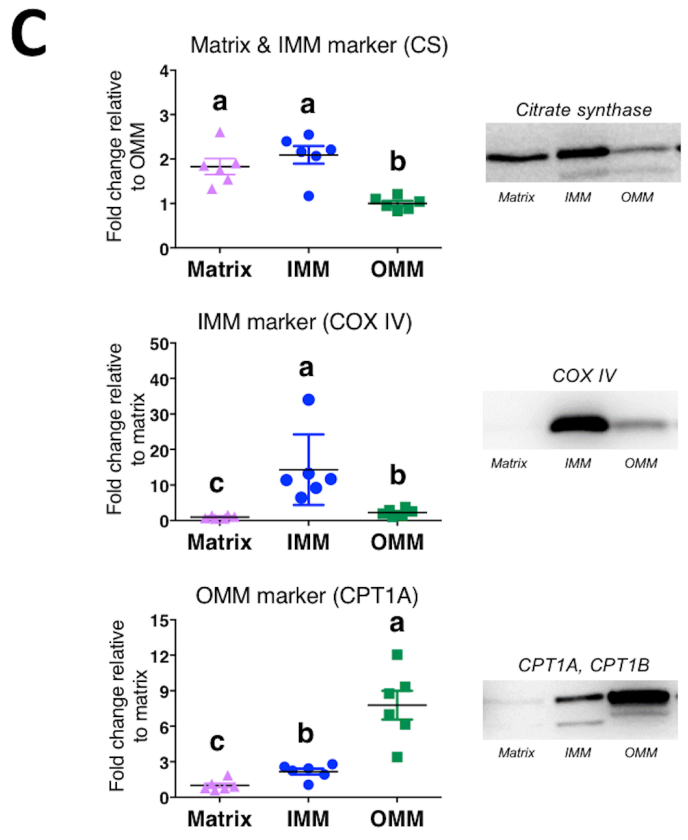

Fig 3 


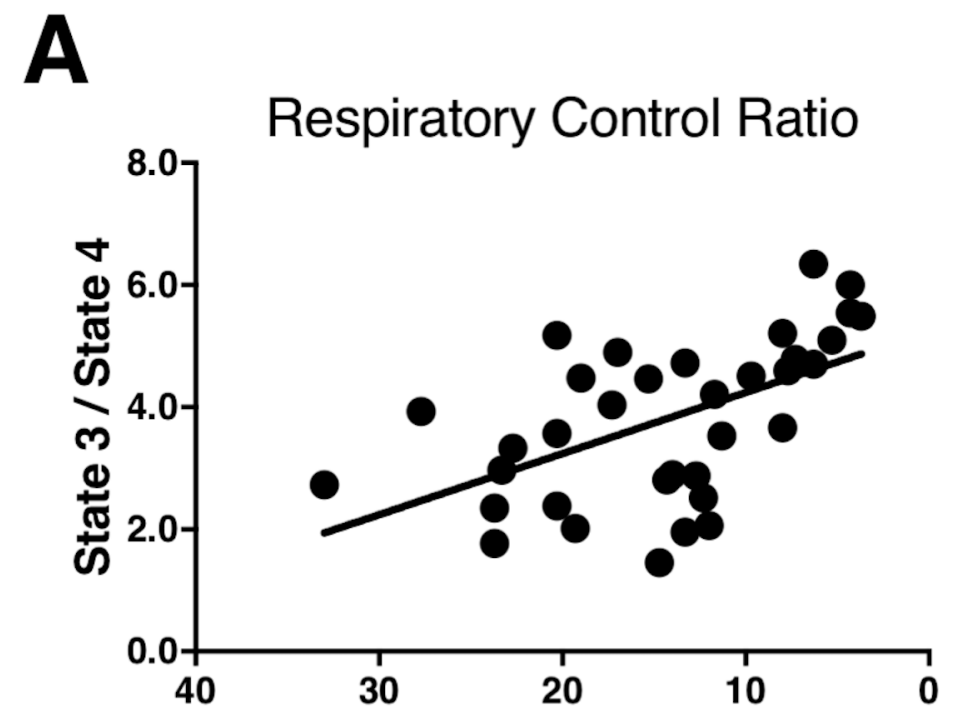

B

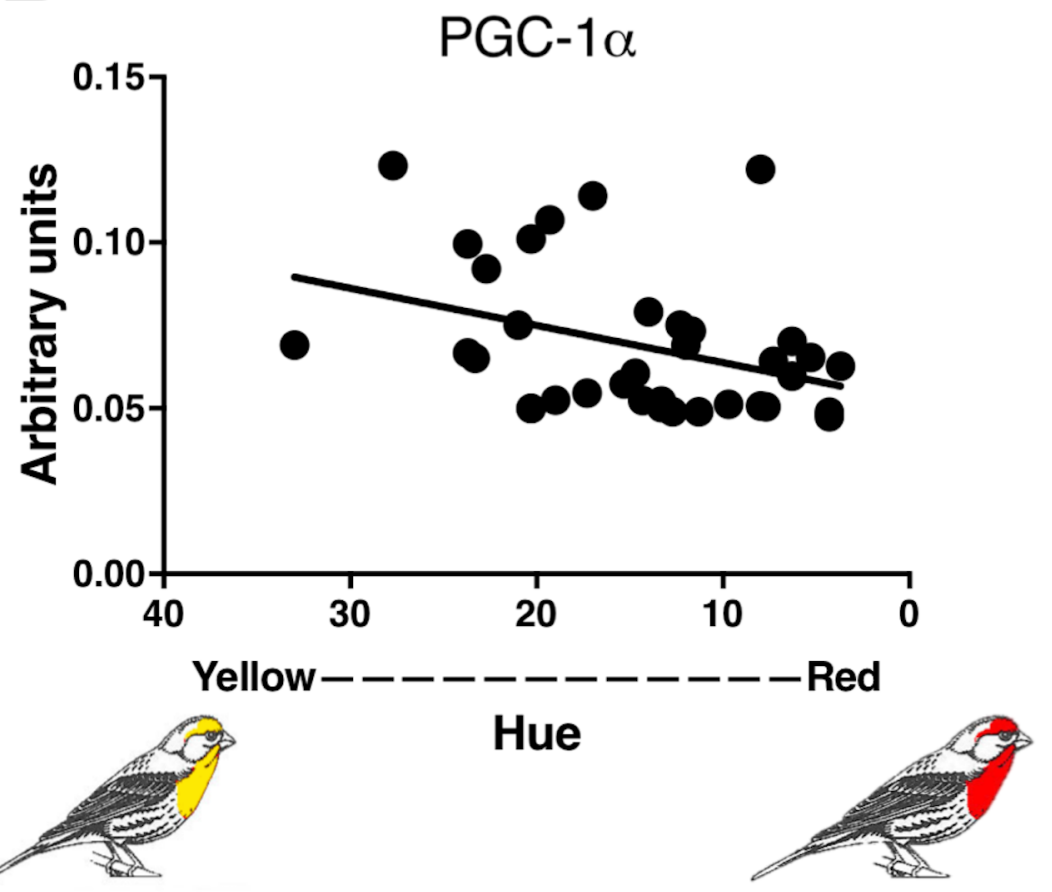




\section{Fig 5}
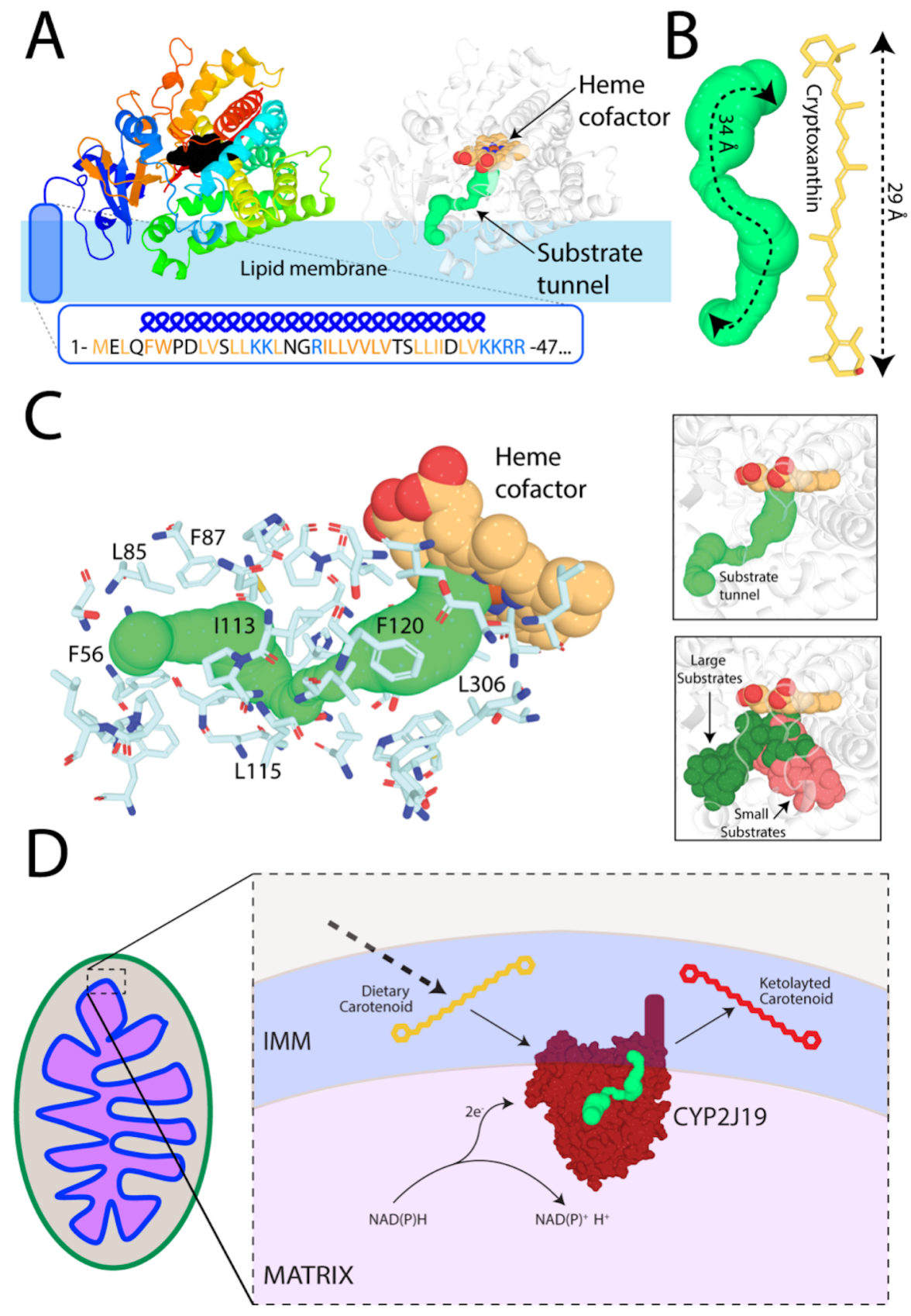
521

522

523

524

525

526

527

528

529

530

531

532

533

534

535

536

537

538

539

540

541

542

543

\section{References}

1. Hill GE. 2007 Melanins and carotenoids as feather colorants and signals. In Reproductive Biology and Phylogeny of Birds: Sexual Selection, Behavior, Conservation, Embryology and Genetics, Vol 6B. (ed BGM Jamieson), pp. 41-74. Enfield, NH: Science Publishers.

2. Pryke SR, Andersson S, Lawes MJ, Piper SE. 2002 Carotenoid status signaling in captive and wild red-collared widowbirds: Independent effects of badge size and color. Behav. Ecol. 13, 622-631. (doi:10.1093/beheco/13.5.622)

3. Svensson PA, Wong BBM. 2011 Carotenoid-based signals in behavioural ecology: a review. Behaviour 148, 131-189. (doi:10.1163/000579510X548673)

4. Hill GE. 2006 Female mate choice for ornamental coloration. Bird Color. Vol. II. Funct. Evol. , 137-200.

5. Hill GE. 2006 Environmental Regulation of Ornamental Coloration. In Bird Coloration: Vol. 1 Mechanisms and Measurements (eds GE Hill, KJ McGraw), pp. 507-560. Cambridge MA: Harvard University Press.

6. Blount J, McGraw K. 2008 Signal functions of carotenoid colouration. In Carotenoids (eds S Liaaen-Jensen, H Pfander), pp. 213-236. Basel, Switzerland: Birkhäuser. (doi:10.1007/978-3-7643-7499-0)

7. Weaver RJ, Santos ESA, Tucker AM, Wilson AE, Hill GE. 2018 Carotenoid metabolism strengthens the link between feather coloration and individual quality. Nat. Commun. 9, 73. (doi:10.1038/s41467-017-02649-z)

8. McGraw KJ, Ardia DR. 2003 Carotenoids, immunocompetence, and the information content of sexual colors: an experimental test. Am. Nat. 162, 704-712.

9. Hill GE. 2014 Cellular respiration: the nexus of stress, condition, and ornamentation. 
Integr. Comp. Biol. 54, 645-657. (doi:10.1093/icb/icu029)

10. Pike TW, Blount JD, Bjerkeng B, Lindström J, Metcalfe NB. 2007 Carotenoids, oxidative stress and female mating preference for longer lived males. Proc. R. Soc. London B Biol. Sci. 274, 1591-1596.

11. Perez-Rodriguez L, Mougeot F, Alonso-Alvarez C. 2010 Carotenoid-based coloration predicts resistance to oxidative damage during immune challenge. J. Exp. Biol. 213, 1685-1690. (doi:10.1242/jeb.039982)

12. Koch RE, Hill GE. 2018 Do carotenoid-based ornaments entail resource tradeoffs? An evaluation of theory and data. Funct. Ecol. 32, 1908-1920. (doi:https://doi.org/10.1111/1365-2435.13122)

13. Weaver RJ, Koch RE, Hill GE. 2017 What maintains signal honesty in animal colour displays used in mate choice? Philos. Trans. R. Soc. B Biol. Sci. 372, 20160343. (doi:10.1098/rstb.2016.0343)

14. Garratt M, Brooks RC. 2012 Oxidative stress and condition-dependent sexual signals: more than just seeing red. Proc. R. Soc. B Biol. Sci. 279, 3121-3130. (doi:10.1098/rspb.2012.0568)

15. Simons MJP, Cohen AA, Verhulst S. 2012 What does carotenoid-dependent coloration tell? Plasma carotenoid level signals immunocompetence and oxidative stress state in birds-a meta-analysis. PLoS One 7, e43088. (doi:10.1371/journal.pone.0043088)

16. Alonso-Alvarez C, Pérez-Rodríguez L, Mateo R, Chastel O, Viñuela J. 2008 The oxidation handicap hypothesis and the carotenoid allocation trade-off. J. Evol. Biol. 21, 1789-1797. (doi:10.1111/j.1420-9101.2008.01591.x)

17. Møller AP, Biard C, Blount JD, Houston DC, Ninni P, Saino N, Surai PF. 2000 
Carotenoid-dependent Signals: Indicators of Foraging Efficiency, Immunocompetence or

Detoxification Ability? Avian Poult. Biol. Rev. 11, 137-159.

569

570

571

572

573

574

575

576

577

578

579

580

581

582

583

584

585

586

587

588

589

18. Costantini D, Møller AP. 2008 Carotenoids are minor antioxidants for birds. Funct. Ecol.

22, 367-370. (doi:10.1111/j.1365-2435.2007.01366.x)

19. Hill GE, Johnson JD. 2012 The vitamin A-redox hypothesis: a biochemical basis for honest signaling via carotenoid pigmentation. Am. Nat. 180, E127--50.

(doi:10.1086/667861)

20. Mcgraw KJ. 2006 Mechanics of carotenoid coloration. In Bird Coloration, Volume 1: Measurements and Mechanisms (eds GE Hill, KJ McGraw), pp. 177-242. Cambridge MA: Harvard University Press, Cambridge, Mass.

21. Lopes RJ et al. 2016 Genetic Basis for Red Coloration in Birds. Curr. Biol. 26, 14271434. (doi:10.1016/j.cub.2016.03.076)

22. Mundy NII et al. 2016 Red Carotenoid Coloration in the Zebra Finch Is Controlled by a Cytochrome P450 Gene Cluster. Curr. Biol. 26, 1435-1440.

(doi:10.1016/j.cub.2016.04.047)

23. Twyman H, Andersson S, Mundy NI. 2018 Evolution of CYP2J19, a gene involved in colour vision and red coloration in birds: positive selection in the face of conservation and pleiotropy. BMC Evol. Biol. 18, 22.

24. Inouye CY, Hill GE, Montgomerie R. 2012 Carotenoid pigments in male House Finch plumage in relation to age, subspecies, and ornamental coloration. Auk 118, 900-915. (doi:10.2307/4089841)

25. Johnson JD, Hill GE. 2013 Is carotenoid ornamentation linked to the inner mitochondria membrane potential? A hypothesis for the maintenance of signal honesty. Biochimie $\mathbf{9 5}$, 

436-444. (doi:10.1016/j.biochi.2012.10.021)

26. Hill GE. 2002 A red bird in a brown bag: the function and evolution of ornamental plumage coloration in the House Finch. New York: Oxford University Press.

27. McKay BD. 2013 The use of digital photography in systematics. Biol. J. Linn. Soc. 110, $1-13$.

28. Hill GE, Hood WR, Huggins K. 2009 A multifactorial test of the effects of carotenoid access, food intake and parasite load on the production of ornamental feathers and bill coloration in American goldfinches. J. Exp. Biol. 212, 1225-1233.

(doi:10.1242/jeb.026963)

29. Trounce IA, Kim YL, Jun AS, Wallace DC. 1996 Assessment of mitochondrial oxidative phosphorylation in patient muscle biopsies, lymphoblasts, and transmitochondrial cell lines. Methods Enzymol. 264, 484-509. (doi:10.1016/S0076-6879(96)64044-0)

30. Palczewski G, Amengual J, Hoppel CL, Von Lintig J. 2014 Evidence for compartmentalization of mammalian carotenoid metabolism. FASEB J. 28, 4457-4469. (doi:10.1096/fj.14-252411)

31. Zhang Y, Humes F, Almond G, Kavazis A, Hood WR. 2017 A mitohormetic response to pro-oxidant exposure in the house mouse. Am. J. Physiol. Integr. Comp. Physiol. 314, R122-R134. (doi:10.1152/ajpregu.00176.2017)

32. Lambert AJ, Buckingham JA, Boysen HM, Brand MD. 2008 Diphenyleneiodonium acutely inhibits reactive oxygen species production by mitochondrial complex I during reverse, but not forward electron transport. Biochim. Biophys. Acta - Bioenerg. 1777, 397403. (doi:10.1016/j.bbabio.2008.03.005)

33. Åkerman KEO, Wikström MKF. 1976 Safranine as a probe of the mitochondrial 
membrane potential. FEBS Lett. 68, 191-197. (doi:10.1016/0014-5793(76)80434-6)

34. Miwa S, Treumann A, Bell A, Vistoli G, Nelson G, Hay S, Von Zglinicki T. 2016 Carboxylesterase converts Amplex red to resorufin: Implications for mitochondrial H2O2release assays. Free Radic. Biol. Med. 90, 173-183. (doi:10.1016/j.freeradbiomed.2015.11.011)

35. R Core Team. $2014 R$ : A language and environment for statistical computing. Vienna, Austria: Foundation for Statistical Computing.

36. Kim DE, Chivian D, Baker D. 2004 Protein structure prediction and analysis using the Robetta server. Nucleic Acids Res. 32, W526-W531. (doi:10.1093/nar/gkh468)

37. Roy A, Kucukural A, Zhang Y. 2010 I-TASSER: A unified platform for automated protein structure and function prediction. Nat. Protoc. 5, 725. (doi:10.1038/nprot.2010.5)

38. Chovancova E et al. 2012 CAVER 3.0: A Tool for the Analysis of Transport Pathways in Dynamic Protein Structures. PLoS Comput. Biol. 10, e1002708. (doi:10.1371/journal.pcbi.1002708)

39. Ge Z, Johnson JD, Cobine PA, McGraw KJ, Garcia R, Hill GE. 2015 High concentrations of ketocarotenoids in hepatic mitochondria of Haemorhous mexicanus. Physiol. Biochem. Zool. 88, 444-450. (doi:10.1086/681992)

40. Amengual J, Lobo GP, Golczak M, Li HNM, Klimova T, Hoppel CL, Wyss A, Palczewski K, von Lintig J. 2011 A mitochondrial enzyme degrades carotenoids and protects against oxidative stress. FASEB J. 25, 948-959. (doi:10.1096/fj.10-173906)

41. Lobo GP, Isken A, Hoff S, Babino D, von Lintig J. 2012 BCDO2 acts as a carotenoid scavenger and gatekeeper for the mitochondrial apoptotic pathway. Development 139, 2966-2977. (doi:10.1242/dev.079632) 
636

637

638

639

640

641

642

643

644

645

646

647

648

649

650

651

652

653

654

655

656

657

658

42. Galván I, Garrido-Fernández J, Ríos J, Pérez-Gálvez A, Rodríguez-Herrera B, Negro JJ. 2016 Tropical bat as mammalian model for skin carotenoid metabolism. Proc. Natl. Acad. Sci. 113, 10932-10937. (doi:10.1073/pnas.1609724113)

43. Barja G. 1998 Mitochondrial free radical production and aging in mammals and birds. Ann. N. Y. Acad. Sci. 854, 224-238. (doi:10.1111/j.1749-6632.1998.tb09905.x)

44. Barja G. 2013 Updating the Mitochondrial Free Radical Theory of Aging: An Integrated View, Key Aspects, and Confounding Concepts. Antioxid. Redox Signal. 19, 1420-1445. (doi:10.1089/ars.2012.5148)

45. Porter C et al. 2015 Mitochondrial respiratory capacity and coupling control decline with age in human skeletal muscle. Am. J. Physiol. - Endocrinol. Metab. 309, E224-E232. (doi:10.1152/ajpendo.00125.2015)

46. Cawthon D, McNew R, Beers KW, Bottje WG. 1999 Evidence of mitochondrial dysfunction in broilers with pulmonary hypertension syndrome (ascites): Effect of t-butyl hydroperoxide on hepatic mitochondrial function, glutathione, and related thiols. Poult. Sci. 78, 114-124. (doi:10.1093/ps/78.1.114)

47. Austin S, St-Pierre J. 2012 PGC1 alpha and mitochondrial metabolism - emerging concepts and relevance in ageing and neurodegenerative disorders. J. Cell Sci. 125, 49634971. (doi:10.1242/jcs.113662)

48. Dröge W. 2002 Free radicals in the physiological control of cell function. Physiol. Rev. 82, 47-95. (doi:10.1152/physrev.00018.2001)

49. Ray PD, Huang B-W, Tsuji Y. 2012 Reactive oxygen species (ROS) homeostasis and redox regulation in cellular signaling. Cell. Signal. 24, 981-990.

(doi:10.1016/J.CELLSIG.2012.01.008) 
50. Schieber M, Chandel NSS. 2014 ROS function in redox signaling and oxidative stress. Curr. Biol. 24, R453-R462. (doi:10.1016/J.CUB.2014.03.034)

51. Hood WR, Zhang Y, Mowry A V, Hyatt HW, Kavazis AN. 2018 Life History Trade-offs within the Context of Mitochondrial Hormesis. Integr. Comp. Biol. 58, 567-577. (doi:10.1093/icb/icy073)

52. Hill GE. 1991 Plumage coloration is a sexually selected indicator of male quality. Nature 350, 337-339. (doi:10.1038/350337a0)

53. Hill GE, Farmer KL. 2005 Carotenoid-based plumage coloration predicts resistance to a novel parasite in the house finch. Naturwissenschaften 92, 30-34. (doi:10.1007/s00114004-0582-0)

54. Braun EJ, Sweazea KL. 2008 Glucose regulation in birds. Comp. Biochem. Physiol. - B Biochem. Mol. Biol. 151, 1-9. (doi:10.1016/j.cbpb.2008.05.007)

55. Kuzmiak S, Glancy B, Sweazea KL, Willis WT. 2012 Mitochondrial function in sparrow pectoralis muscle. J. Exp. Biol. 215, 2039-2050. (doi:10.1242/jeb.065094)

56. McWilliams SR, Guglielmo C, Pierce B, Klaassen M. 2004 Flying, fasting, and feeding in birds during migration: a nutritional and physiological ecology perspective. J. Avian Biol. 35, 377-393.

57. Twyman H, Valenzuela N, Literman R, Andersson S, Mundy NI, Mundy NI. 2016 Seeing red to being red : conserved genetic mechanism for red cone oil droplets and co-option for red coloration in birds and turtles. Proc. R. Soc. London. Ser. B Biol. Sci. 283, 20161208. (doi:10.1098/rspb.2016.1208)

58. S Šrejber M, Navrátilová V, Paloncýová M, Bazgier V, Berka K, Anzenbacher P, Otyepka M. 2018 Membrane-attached mammalian cytochromes P450: An overview of the 
682

683

684

685

686

687

membrane's effects on structure, drug binding, and interactions with redox partners. $J$.

Inorg. Biochem. 183, 117-136. (doi:10.1016/j.jinorgbio.2018.03.002)

59. Cojocaru V, Winn PJ, Wade RC. 2007 The ins and outs of cytochrome P450s. Biochim.

Biophys. Acta - Gen. Subj. 1770, 390-401. (doi:10.1016/j.bbagen.2006.07.005) 